\title{
Short- and Long-Term Prognosis among Veterans with Neurological Disorders and Subsequent Lower-Extremity Amputation
}

\author{
Janet A. Prvu-Bettger ${ }^{a}$ Barbara E. Bates ${ }^{b}$ Douglas E. Bidelspach ${ }^{c}$ \\ Margaret G. Stineman ${ }^{a}$ \\ a Department of Physical Medicine and Rehabilitation, University of Pennsylvania, Philadelphia, Pa., \\ ${ }^{b}$ Veterans Affairs Medical Center Albany and Albany Medical College, Albany, N.Y., and 'Veterans Affairs \\ Medical Center Lebanon, Lebanon, Pa., USA
}

\section{Key Words}

Amputation • Neurological disorders • United States

veterans $\cdot$ Comorbidity $\cdot$ Survival

\begin{abstract}
Background: Although comorbid neurological conditions are not uncommon for individuals undergoing lower-extremity (LE) amputation, short- and long-term prognosis is unclear. Methods: This cohort study on the survival of United States veterans with LE amputations examined the association between different preexisting neurological conditions and short- and long-term (in-hospital and within 1-year of surgical amputation) mortality. $\chi^{2}$ and t test statistics compared baseline characteristics for patients with and without neurological disorders. Multiple logistic regression and Cox proportional hazard models were used to examine shortand long-term survival and identify predictors limited to the subset of those with neurological conditions adjusting for age, amputation level and etiology, and co-morbidities. $\boldsymbol{R e}$ sults: Of 4,720 patients, $43.3 \%$ had neurological disorders documented. Most prevalent were stroke or hemiparesis (18.3\%) and peripheral nervous system (PNS) disorders (20.3\%). Among patients with neurological conditions, those with a PNS disorder or spinal cord injury (or paralysis) were significantly less likely to die in hospital and within 1 year $(p<0.05)$ when compared to the other types of neurological
\end{abstract}

condition groups including stroke (or hemiparesis), cerebral degenerative diseases, movement disorders and autonomic disorders. Conclusions: The high prevalence of preexisting neurological disorders among LE amputees and the varying effect of different conditions on risk of mortality highlights the need to further characterize the diverseness of this understudied subpopulation. While preexisting spinal cord injury and PNS disorders appear to carry a decreased risk among amputees, those with central nervous system disorders have comparatively greater mortalities.

Copyright $\odot 2008$ S. Karger AG, Basel

\section{Introduction}

Neurological disorders can be quite diverse, challenging to treat and often disabling. Conditions that involve the central nervous system (CNS), peripheral nervous system (PNS) and autonomic nervous system (ANS) are not uncommon in patients who undergo lower-extremity (LE) amputation, and the impact of these conditions on prognosis has not been examined. Understanding the prevalence of neurological disorders that are present as comorbidities prior to LE amputation and the effect on patient outcomes may aid in assessing resource use and quality of care.

\section{KARGER}

() 2008 S. Karger AG, Basel

Fax +41613061234 E-Mail karger@karger.ch www.karger.com
Accessible online at: www.karger.com/ned
Janet Prvu Bettger, ScD

Department of Physical Medicine and Rehabilitation, School of Medicine University of Pennsylvania, New Courtland Center for Transitions and Health 3615 Chestnut Street, 334 Ralston-Penn Center, Philadelphia, PA 19104 (USA)

Tel. +1 617901 0277, Fax +1 215573 2304, E-Mail janetpr@nursing.upenn.edu 
Studies of patients with LE amputations in the United States Veterans Health Administration (VHA) found unadjusted 1-year survival rates of approximately $70 \%$ with poorer rates for older patients and those with a more proximal amputation, renal, cardiovascular or cerebral vascular diseases [1-3]. Those patients categorized as having 'other neurological conditions' had a threefold increased likelihood of in-hospital mortality, second highest only to those with systemic sepsis [3]. Although LE amputees with preexisting neurological conditions have been shown to be at risk for poorer outcomes, work to date using data from the VHA, US private sector, or abroad has not explored the full range of neurological disorders, and neither the prevalence of different types nor their effect on survival is known. The aim of this study was to expand on previous research and determine the prevalence of neurological disorders present as preexisting comorbidities among veterans with LE amputations in order to investigate different conditions as independent predictors of short- (in-hospital) and long-term (1-year) mortality. Recognizing that the vascular system is generally severely compromised in patients with preexisting stroke or hemiparesis, it was hypothesized that this group would be at increased odds of death within 1 year compared to those with other types of neurological conditions. We further hypothesized that patients with spinal cord injury (SCI) or paralysis would be at increased odds of survival to 1 year following the amputation. Those with SCI or paralysis may have already adapted to life with reduced limb use and would logically have a lower incidence of vascular compromise. We were unable to identify any studies of individuals with SCI or paralysis and subsequent amputation and, thus, propose this scenario based on clinical plausibility.

\section{Materials and Methods}

This retrospective longitudinal study was approved by Institutional Review Boards at the University of Pennsylvania and the Veterans Affairs Medical Centers in Albany, New York and Kansas City, Mo, USA.

Data were obtained from 7 VHA administrative databases used to track health status and health care utilization of veterans. The databases supporting our research included 4 Patient Treatment File (PTF) inpatient data sets, 2 outpatient care files and the Beneficiary Identification Record Locator System (BIRLS) death file [4-6]. The PTF captured the International Classification of Diseases, 9th Revision, Clinical Modification (ICD-9-CM) codes relevant to the entire inpatient hospital stay and identified patients who died during the hospitalization. The outpatient care files have a record for each outpatient visit where a principal diagnosis intended to describe the reason for the visit is coded and 9 additional variables are available for secondary diagnostic information. The BIRLS database contained records of all beneficiaries, including veterans whose survivors applied for death benefits. Our methods of data abstraction, identification and exclusion of patients have been described previously [3].

All veterans with new transtibial, transfemoral or hip disarticulation amputations with discharge dates between October 1, 2002, and September 30, 2004, were included. There were 125 US Veterans Affairs Medical Centers operating during this study period but surgical amputation of the lower limbs occurred at only 101 of those facilities. Of the 4,727 patients in this sample population, 7 (0.15\%) were excluded from analyses because of lack of information (6 had an unspecified amputation level, and 1 was missing living location prior to hospitalization).

Patient level characteristics included age, gender, marital status (married vs. not married), prior living location (home vs. extended care and hospital) and several measures of clinical status. Amputation level distinguished between transtibial and transfemoral limb loss. The latter group included patients with hip disarticulations because of low prevalence. Length of acute hospital stay (in days) approximated patient complexity. Using the ICD-9CM codes from outpatient files 3 months prior to hospital admission and documented in the PTF between admission and surgical dates, we differentiated among diagnoses likely to have contributed directly to the amputation as etiologies and concurrent conditions less likely to be related but present as comorbidities. Comorbidities were expressed with the Elixhauser Comorbidity Measure, which includes 30 categories [7]. Neurological comorbidities assessed with the Elixhauser Comorbidity Measure (other neurological comorbidities and paralysis) were not included in analyses. Categories with insufficient prevalence were combined where possible or excluded from analyses. These included obesity (no cases had this condition documented), combined hypertension and hypertension with complications (low prevalence in the latter), and combined cancer categories of solid tumor without metastasis, metastatic cancer and lymphoma (low prevalence in the latter two categories). Peripheral vascular disease, diabetes mellitus and diabetes with complications are instead accounted for as possible etiologies under peripheral circulation problems, and type I and II diabetes mellitus rather than as comorbid conditions. The categorization of the ICD-9-CM codes into 12 etiological conditions, described previously, included congenital deformity, chronic osteomyelitis, device infection, type I and II diabetes mellitus, local significant infection, lower-limb cancer, peripheral circulation problems, previous amputation complication, skin breakdown, systemic sepsis and trauma [3]. Etiologies were viewed as multifactorial, meaning that an individual could have more than one factor contributing to limb loss, just as more than one comorbidity could be present at the time of the amputation.

\section{Neurological Conditions}

Using ICD-9-CM codes documented for each patient, we identified all neurological disorders. The 37 disorders were clinically reviewed and categorized into 7 conditions including a group for 'other' CNS conditions with low prevalence in this sample (ICD9-CM codes in parentheses): 
(1) stroke or hemiparesis as defined by the VHA (342, 430438.9);

(2) cerebral degenerative diseases, i.e. Alzheimer's disease and Reye's syndrome (331);

(3) movement disorders, i.e. Parkinson's disease and ataxias (332334);

(4) ANS disorders including peripheral autonomic neuropathies, reflex sympathetic dystrophies and autonomic dysreflexia (337);

(5) SCI or paralysis including fracture of the vertebral column with SCI (806), injuries to the nerves and spinal cord (952957), other paralytic syndromes (344) and other diseases of the spinal cord (336);

(6) PNS disorders including nerve root and plexus disorders, mononeuritis, peripheral, inflammatory and toxic neuropathies, myoneural disorders, muscular dystrophies, other myopathies and disorders of the cranial nerves (but excluding facial nerves) (352-359);

(7) 'other' CNS conditions including brain injury as defined by the VHA (800-801, 803-804, 850-854.19, 950-951, 959.01), inflammatory or infectious diseases such as meningitis (320$326)$, other conditions of the brain (348), anterior horn cell disease (335), multiple sclerosis and other demyelinating diseases of the CNS (340-341). (No patients had ICD-9 codes $345-347$ to indicate epilepsy, migraines, cataplexy or narcolepsy.)

\section{Outcome}

Patients were followed from the surgical date through the 1year anniversary of their surgical amputation. Death during the acute hospitalization (short-term prognosis), death within 1 year of the amputation (long-term prognosis) and number of days between surgery and death were examined. Mortality information was acquired from the PTF and BIRLS databases. Combining the BIRLS with the PTF provides a thorough list of the veterans with LE amputations who died. Neither the PTF nor the BIRLS provide specific cause of death information as indicated by an ICD-9-CM code.

The main PTF database was accessed first, since it identifies patients who died in a VHA hospital. If a death date was documented on or prior to discharge, that date was used. The BIRLS database, documented to have a sensitivity of 70-96.5\% [8], was then examined for the remaining patients who did not die in a VHA hospital to determine if the death date followed the discharge date. If no death date was found in either database, it was assumed that the veteran was still alive 1 year following the amputation.

\section{Statistical Analysis}

The distribution of neurological disorders was examined to identify the proportion of patients with evidence of each type. Patients who had 2 or more neurological disorders were reported in multiple categories, but the number of patients with neurological conditions was determined independently of the number of coexisting disorders. Baseline patient characteristics were compared for patients with and without neurological disorders using $\chi^{2}$ and t test statistics. The mortality rate for patients with neurological disorders was calculated and reported as the unadjusted rate. To address the relative associations between each type of condition and mortality, three regression models were created in the subsample of patients with neurological conditions. Multiple logistic regression with forward selection was used to identify the patient characteristics predictive of short- and longerterm mortality (dichotomous dependent variables for death during the hospital stay and within 1 year of the amputation, respectively). All patient characteristics were eligible and were allowed to enter each model when $\mathrm{p}<0.05$. A Cox proportional hazards model using backward selection (removing variables when $p>0.05$ ) was then used to determine the hazard ratio for the likelihood of amputees with a neurological disorder surviving 1 year after their amputation. This highly saturated model included all patient characteristics in order to examine the simultaneous effects of multiple variables that could be influencing prognosis. A Kaplan-Meier survival plot was created to present cumulative survival by the number of days from surgery, adjusting for statistically significant patient characteristics that were identified in the Cox regression model.

\section{Results}

Of this primarily male sample of veterans ranging in age from 26 to 107 years (mean age 67.4, SD = 10.7), 43.3\% had a neurological disorder documented prior to the amputation. It was significantly more common for patients with neurological disorders, compared to those without, to be married and have a longer length of hospital stay. Fewer lived at home prior to the hospitalization or had the amputation due to a device infection, yet more had an etiology of local significant infection, type I or II diabetes mellitus, or skin breakdown. Several comorbid conditions were significantly more prevalent in amputees with neurological disorders including congestive heart failure, deficiency anemias, depression, fluid and electrolyte disturbances, hypertension and psychoses; yet, it was less common to have comorbid cancer or rheumatoid arthritis. There was no difference between the groups in age, gender or amputation level (table 1).

\section{Subsample of LE Amputees with Preexisting \\ Neurological Disorders $(n=2,042)$}

Seven neurological condition categories were examined. Most prevalent were PNS disorders $(\mathrm{n}=957 ; 42.3 \%)$ and stroke/hemiparesis $(864 ; 38.2 \%)$. A similar proportion of patients had SCI/paralysis $(197 ; 8.7 \%)$ or an ANS disorder $(166 ; 7.3 \%)$. Fewer patients had a cerebral degenerative disease $(104 ; 4.6 \%)$, movement disorder $(102 ; 4.5 \%)$ or 'other' conditions $(84 ; 3.7 \%)$. Patients who had 2 or more neurological conditions documented are reported in multiple categories. There were 393 patients $(8.3 \%$ of the full sample; $19.2 \%$ of the neurological condition subsample) with 2 or more neurological conditions. Unadjusted in-hospital survival for the 2,042 patients with 
Table 1. Unadjusted characteristics of veterans with amputations who did and did not have a neurological disorder

\begin{tabular}{lccc}
\hline Patient characteristics & $\begin{array}{c}\text { With a neurological } \\
\text { disorder }(\mathrm{n}=2,042)\end{array}$ & $\begin{array}{c}\text { Without a neurological } \\
\text { disorder }(\mathrm{n}=2,678)\end{array}$ & p value \\
\hline Mean age, years & $66.9 \pm 11.0$ & $66.9 \pm 11.3$ & 0.904 \\
Male gender & $2,025(99.2)$ & $2,654(99.1)$ & 0.815 \\
Married & $1,004(49.2)$ & $1,128(42.1)$ & $<0.0001$ \\
Living at home prior to hospitalization & $1,732(84.8)$ & $2,330(87.0)$ & 0.046 \\
Transfemoral level of amputation & $821(40.2)$ & $1,031(38.5)$ & 0.148 \\
Mean length of hospital stay, days & $33.7 \pm 90.5$ & $26.2 \pm 31.1$ & 0.005 \\
Etiology & & & $<0.0001$ \\
$\quad$ Device infection & $178(8.7)$ & $352(13.1)$ & 0.0003 \\
Diabetes mellitus type I & $365(17.9)$ & $375(14.0)$ & $<0.0001$ \\
Diabetes mellitus type II & $1,499(73.4)$ & $1,656(61.8)$ & 0.002 \\
Local significant infection & $1,608(78.8)$ & $1,006(74.9)$ & $<0.0001$ \\
Skin breakdown & $1,459(71.5)$ & $276(60.5)$ & 0.001 \\
Nonneurological comorbidities & $155(7.6)$ & $598(22.3)$ & 0.006 \\
Cancer & $526(25.8)$ & $580(21.7)$ & 0.030 \\
Congestive heart failure & $497(24.3)$ & $225(8.4)$ & 0.003 \\
$\quad$ Deficiency anemias & $224(11.0)$ & $549(20.5)$ & 0.001 \\
Depression & $501(24.5)$ & $1,613(60.2)$ & 0.003 \\
Fluid and electrolyte disturbances & $1,318(64.5)$ & $170(6.4)$ & 0.030 \\
Hypertension & $163(8.0)$ & $46(1.7)$ & 0.022 \\
Psychoses & $19(0.9)$ & & \\
Rheumatoid arthritis & & & \\
\hline
\end{tabular}

Data are expressed as numbers, with percentages in parentheses, or means \pm standard deviation. Patient characteristics were compared for patients with and without neurological disorders using $\chi^{2}$ and test statistics for categorical and continuous variables, respectively. Only etiologies and comorbidities significantly different between those with and without neurological disorders are presented in the table. Thus, descriptive statistics for 4 etiologies and 16 comorbidities are not presented.

neurological disorders was $93.4 \%$, and 1-year survival was $76.3 \%$.

Among those with neurological conditions, patients with SCI/paralysis or PNS disorders were less likely to die in hospital than patients in the remaining 5 categories of neurological conditions ( $\mathrm{SCI} /$ paralysis odds ratio, $\mathrm{OR}=$ $0.37,95 \%$ confidence interval, $\mathrm{CI}=0.15-0.87, \mathrm{p}=0.023$; $\mathrm{PNS}$ OR $=0.44,95 \% \mathrm{CI}=0.25-0.78, \mathrm{p}=0.0005)$. The same was true for death within 1 year $(\mathrm{SCI} /$ paralysis $\mathrm{OR}=0.56,95 \% \mathrm{CI}=0.35-0.90, \mathrm{p}=0.017$; PNS OR $=0.58$, $95 \% \mathrm{CI}=0.42-0.80, \mathrm{p}=0.0007)$, whereas patients with 'other' conditions were more likely to die within 1 year ('other' OR $=1.86,95 \% \mathrm{CI}=1.10-3.14, \mathrm{p}=0.021$ ). Significant predictors of both death in hospital and death at 1 year were older age, transfemoral amputation, systemic sepsis etiology, and comorbid arrhythmia, renal failure, and fluid and electrolyte disturbances. Unique predictors of death in hospital included prior living location other than home and the absence of chronic osteomyelitis as an etiology. Unique predictors of death at 1 year were comorbid cancer, chronic pulmonary disease, congestive heart failure, valvular disease and weight loss.

The 1-year survival estimates were similar in the Cox regression after adjusting for all patient characteristics (table 2). Of the 7 neurological conditions examined, patients with SCI/paralysis were least likely to die within 1 year (hazard ratio $=0.54, \mathrm{p}=0.003$ ), as were those with PNS disorders (hazard ratio $=0.62, \mathrm{p}=0.0003$ ), when compared to patients in the remaining neurological condition categories. There was no statistically significant difference in survival for patients with stroke/hemiparesis, ANS disorders, cerebral degenerative diseases or movement disorders ( $\mathrm{p}>0.05)$ when compared to the remaining neurological categories. Significant predictors of 1-year mortality for this neu rological-amputation population included older age, transfemoral amputation, systemic sepsis etiology, and comorbid arrhythmia, cancer, chronic pulmonary disease, fluid and electrolyte distur- 
Table 2. Cox proportional hazard analysis $(n=2,042)$

\begin{tabular}{|c|c|c|c|c|}
\hline Variable & $\begin{array}{l}\text { Percent dead } \\
\text { at } 1 \text { year }\end{array}$ & Hazard ratio & $95 \% \mathrm{CI}$ & $\mathrm{p}$ value \\
\hline Neurological conditions & 23.7 & & & \\
\hline Peripheral nervous system disorder & 15.3 & 0.62 & $0.48-0.80$ & 0.0003 \\
\hline Stroke or hemiparesis & 30.2 & 1.14 & $0.89-1.46$ & 0.315 \\
\hline SCI or paralysis & 13.0 & 0.54 & $0.36-0.81$ & 0.003 \\
\hline Autonomic disorder & 21.5 & 1.07 & $0.75-1.54$ & 0.708 \\
\hline Cerebral degenerative disease & 37.3 & 1.02 & $0.71-1.47$ & 0.922 \\
\hline Movement disorder & 27.1 & 0.94 & $0.62-1.42$ & 0.765 \\
\hline 'Other' (including brain injury) & 32.8 & 1.68 & $1.13-2.52$ & 0.011 \\
\hline \multicolumn{5}{|l|}{ Significant covariates } \\
\hline \multicolumn{5}{|l|}{ Patient factors } \\
\hline Older age & & 1.21 & $1.15-1.27$ & $<0.0001$ \\
\hline Transfemoral amputation & & 1.46 & $1.21-1.76$ & $<0.0001$ \\
\hline Etiology: systemic sepsis & & 2.08 & $1.63-2.66$ & $<0.0001$ \\
\hline \multicolumn{5}{|l|}{ Comorbid conditions } \\
\hline Arrhythmia & & 1.42 & $1.16-1.74$ & 0.0008 \\
\hline Cancer & & 1.46 & $1.10-1.93$ & 0.009 \\
\hline Chronic pulmonary disease & & 1.28 & $1.04-1.58$ & 0.019 \\
\hline Fluid and electrolyte disturbances & & 1.32 & $1.09-1.61$ & 0.005 \\
\hline Renal failure & & 2.04 & $1.66-2.51$ & $<0.0001$ \\
\hline Valvular disease & & 1.51 & $1.09-2.09$ & 0.013 \\
\hline Weight loss & & 1.75 & $1.27-2.41$ & 0.0006 \\
\hline
\end{tabular}

Reference group for each neurological condition category is the remaining sample with neurological disorders.

bances, renal failure, valvular disease and weight loss. The Kaplan-Meier survival plot presents cumulative survival by the number of days from surgery, adjusting for these statistically significant patient characteristics (fig. 1).

\section{Discussion}

This study examined both short- and long-term mortality in a 2-year cohort of veterans with preexisting neurological disorders and subsequent LE amputation in a large integrated US health system. Almost $50 \%$ of the amputees had a coexisting neurological disorder. Health status prior to amputation was poorer for those with preexisting neurological conditions compared to those without, but the unadjusted mortality rates for the population with neurological conditions were not dissimilar to other medically diverse samples [1-3]. Interestingly, although there was no significant difference in in-hospital or 1year mortality between several of the grouped neurological conditions, it appears that patients with SCI/paralysis and PNS disorders had comparatively lower risks and the otherwise undefined group, which contained neurological conditions that presented too infrequently to study, appeared to have the most virulent neurological conditions associated with the greatest risk.

We are unable to compare the prevalence of these neurological conditions to other samples (US or abroad) because research to date on this complex population has focused on the presence of stroke, hemiparesis or SCI, without attention to the growing population of people with, for example, cerebral degenerative disorders or movement disorders. This limitation in the literature may be a result of requiring a large enough sample to capture patients with these less common preexisting conditions that occur, on average, later in life. Even in our larger sample we identified several disorders with too few patients to analyze independently and thus elected to combine them in an 'other' condition group. Although this group was found to be at increased risk of mortality, the wide range in clinical characteristics defining the disorders makes it difficult to hypothesize what led to this finding. 


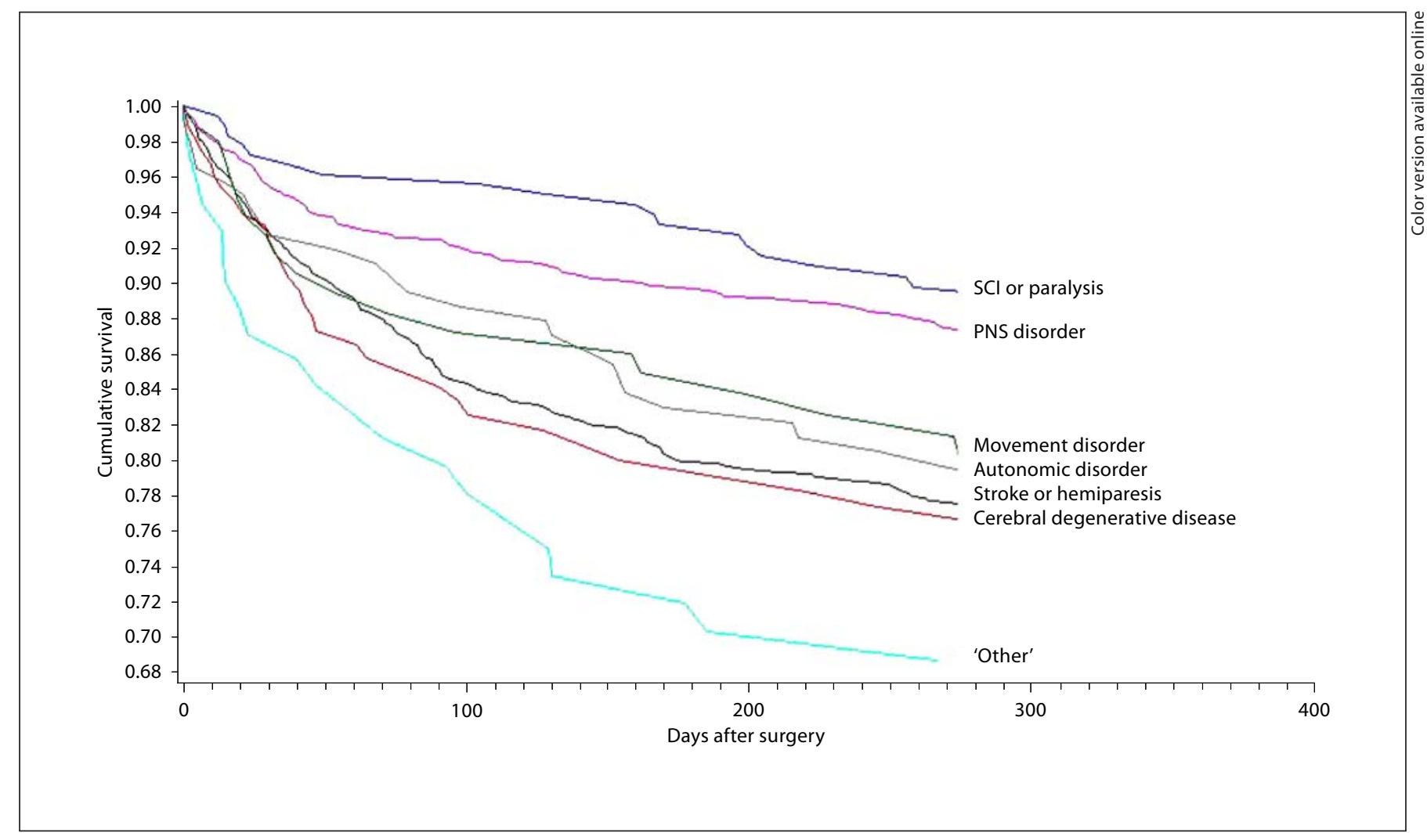

Fig. 1. Cumulative survival plots in days after surgery adjusting for significant patient characteristics; log-rank/ likelihood score test: $\mathrm{p}<0.0001$. Adjusted for patient characteristics significant in the Cox regression: age, amputation level, systemic sepsis etiology, and comorbid arrhythmia, cancer, chronic pulmonary disease, fluid and electrolyte disturbances, renal failure, valvular disease and weight loss.

This study clearly supports that overall health status affects the risk of mortality with neurological disorders being one component of health status in veterans with LE amputations. Age, among other factors, was in fact a stronger predictor of mortality than any of the neurological conditions. Recognizing this, it was thought that the patients with preexisting PNS disorders or SCI/paralysis who were found to have a comparatively lower risk of 1-year mortality could be on average younger. Exploratory analyses of the distribution of age for each of the neurological conditions found no significant differences. Perhaps other clinical characteristics of these groups that are not routinely captured in administrative databases are affecting the mortality risk. Future studies may consider prospectively investigating potential interactions between these neurological conditions and the effects of amputation on mortality, and including measures of severity, disability and health service use both prior to and after surgical amputation as alternate theories.

Neurological Disorders and Prognosis after Amputation
As was expected, one of our largest patient groups included those with stroke or hemiparesis. The literature to date has identified $4-29 \%$ of LE amputees who have had a stroke at some time $[9,10]$, and as was noted by Brunelli et al. [9], it has only been in the last 3 decades that studies have identified factors influencing outcomes for this population. Younger versus older age and transtibial versus transfemoral amputation have been shown to predict more positive rehabilitation outcomes for stroke patients $[9,10]$; the same was true in our study predicting 1-year survival in a more diverse neurological population. In comparison to work done by colleagues [3], this study of patients with neurological disorders identified several patient characteristics and medical comorbidities that seemed to have a similar effect on 1-year mortality (including older age, transfemoral amputation, systemic sepsis etiology, comorbid cancer, fluid and electrolyte disturbances, and renal failure). On the other hand, comorbid arrhythmia, valvular disease and weight loss

Neuroepidemiology 2009;32:4-10 
were uniquely associated with 1-year mortality in this neurological population.

There are many benefits to examining patient care in the VHA, and there are important limitations to acknowledge. A documentation of race was not available for the entire sample and thus was not examined. Veterans are predominately male, and it is unknown if findings can be generalized to females. Studying outcomes following VHA care may not be generalizable to private sector studies. Future research of the VHA population would benefit from including private sector data and exploring the care provided to this complex population.

\section{Conclusions}

The high prevalence of preexisting neurological disorders among LE amputees and the varying effect of different conditions on risk of mortality highlights the diverseness of this understudied population. Additional patient characteristics such as level of disability prior to and following amputation should be considered for inclusion in future studies to help explain the relationship of preexisting neurological conditions with postsurgical outcomes. Health system factors and processes of care should also be considered for inclusion in future studies, particularly as acute rehabilitation has recently been shown to in- crease the odds of survival for LE amputees [11]. Given this important scientific advancement, future studies need to examine the care provided for amputees with compromising preexisting conditions, such as neurological disorders, to ensure there are no disparities in accessing appropriate care for both the amputation and any primary illnesses and impairments. Careful oversight is needed both during acute care and following the hospitalization to ensure that all conditions are appropriately managed.

\section{Acknowledgements}

We would like to thank Dr. Dean M. Reker, PhD, for his clinical expertise in guiding this work and Ms. Sharon Jayne for providing support in the preparation of the manuscript. This material is based upon work supported in part by the National Center for Medical Rehabilitation Research and the National Institutes of Health (RO1-HD042588). It is also supported in part by a Postdoctoral Fellowship for J.A.P.-B. (T32-HD-007425) awarded to the University of Pennsylvania from the National Institute of Child Health and Human Development National Center for Medical Rehabilitation Research. Additional support was provided by resources and the use of facilities at the Samuel S. Stratton Department of Veterans Affairs Medical Center in Albany, N.Y., and the Kansas City Department of Veterans Affairs Medical Center in Kansas City, Mo., USA. The opinions and conclusions of the authors are not necessarily those of the sponsoring agencies.

\section{References}

1 Feinglass J, Pearce WH, Martin GJ, et al: Postoperative and late survival outcomes after major amputation: findings from the Department of Veterans Affairs National Surgical Quality Improvement Program. Surgery 2001;130:21-29.

-2 Mayfield JA, Reiber GE, Maynard C, et al: Survival following lower-limb amputation in a veteran population. J Rehabil Res Dev 2001;38:341-345.

>3 Bates B, Stineman MG, Reker DM, Kurichi JE, Kwong PL: Risk factors associated with mortality in a male veteran population following transtibial or transfemoral amputation. J Rehabil Res Dev 2006;43:917-928.
4 VIReC Research User Guide: FY2000 VHA Medical SAS Inpatient Datasets. Hines, Edward J Hines Jr VA Hospital, Veterans Affairs Information Resource Center, 2003.

5 VIReC Research User Guide: FY2000 VHA Medical SAS Outpatient Datasets. Hines, Edward J Hines Jr VA Hospital, Veterans Affairs Information Resource Center, 2003.

6 Kubal JD, Webber S, Cooper DC, et al: A primer on US mortality databases used in health services research; in VIReC Insights No 5. Hines, Veterans Affairs Information Resource Center, 2000.

7 Elixhauser A, Steiner C, Harris DR, Coffey RM: Comorbidity measures for use with administrative data. Med Care 1998;36:8-27.

$\checkmark 8$ Sohn MW, Arnold N, Maynard C, Hynes D: Accuracy and completeness of mortality data in the Department of Veterans Affairs. Popul Health Metr 2006;4:2. $\checkmark 9$ Brunelli S, Averna T, Porcacchia P, et al: Functional status and factors influencing the rehabilitation outcome of people affected by above-knee amputation and hemiparesis. Arch Phys Med Rehabil 2006;87:995-1000.

10 O'Connell PG, Gnatz S: Hemiparesis and amputation: rehabilitation in the dual disability. Arch Phys Med Rehabil 1989;70:451454.

11 Stineman MG, Kwong PL, Kurichi JE, Prvu Bettger JA, Vogel WB, Maislin G, Bates BE, Reker DM: The effectiveness of inpatient rehabilitation for veterans in the acute postoperative phase of care after trans-tibial or trans-femoral amputation. Arch Phys Med Rehabil 2008, in press. 